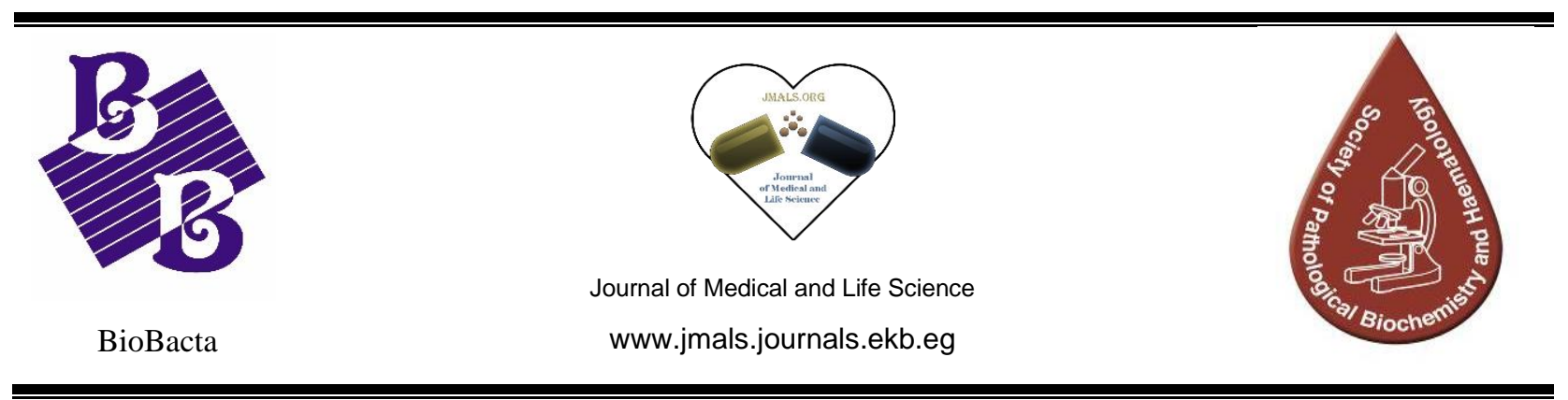

\title{
The correlation between circulating antigen in urine and the intensity of Schistosoma haematobium infection
}

\author{
Ahmed Abdelhalim Yameny \\ Society of Pathological Biochemistry and Hematology, Egypt \\ Ahmed A. Yameny (Email: dr.ahmedyameny@yahoo.com) \\ Received February, 10, 2020; Accepted March, 12, 2020; Published March 15, 2020 \\ DOI: $10.21608 /$ JMALS.2020.111166
}

\begin{abstract}
Background: There are two methods for diagnosis of schistosomiasis, parasitological(microscopic detection of eggs), and/or immunological methods (antibody and antigen detection), due to the decreasing and elimination of Schistosomiasis infection in many countries, detection of Antigen is more valuable for active infection detection, Schistosome antigens are present in serum and urine of infected subjects. Subjects and methods: This study is a cross-sectional study designed to study the relation between a commercially available kit, using circulating antigen in urine and the intensity of infection of S.haematobium detected by microscopic examination in urine, in the study population, this performed on 50 positive S.haematobium samples and 50 negative samples for individuals matching in age and gender, in addition, urine microscopic examination was done for detection of S.haematobium eggs by sedimentation centrifugation and nuclepore filteration techniques. Results: The results showed that the Schistosoma haematobium infection as detected by the Circulating antigen in urine related to the intensity of infection detected by parasitologic techniques. In light cases, it detected $54.5 \%$, and in heavy cases, it increased to $66.7 \%$, While, P-value is 0.575 not significant at $\mathrm{p}<0.05$.
\end{abstract}

Keywords: Schistosoma haematobium, CAg, circulating antigen, intensity, urinary schistosomiasis.

\section{Introduction}

Schistosomiasis infection was decreased in many countries and has been eliminated in Iran, Lebanon, Morocco, and Tunisia with the absence of newly recorded cases in the past few years (WHO, $2007)^{(1)}$. Egypt has started a campaign to reach the final elimination of schistosomiasis by $2020^{\text {(2). }}$ There are two methods for diagnosis of schistosomiasis, parasitological (microscopic detection of eggs), and/or immunological methods (antibody and antigen detection) ${ }^{(3)}$. Questionnaire and chemical reagent strip for haematuria and proteinuria can be considered for the diagnosis of $S$. haematobium where microscopy is unavailable In areas with a high prevalence of infection ${ }^{(4,5)}$, but due to decreasing and elimination of Schistosomiasis infection in many countries, detection of Antigen is more valuable for active 
infection detection, Schistosome antigens are present in serum and urine of infected subjects ${ }^{(6)}$. So many medical laboratories in Egypt using a commercially available kit by circulating antigen (Schistofast ABC Diagnostic), this research aimed the relation between a commercially to study available kit, using circulating antigen in urine and the intensity of infection of S.haematobium detected by microscopic examination in the urine.

\section{Material and methods}

\subsection{Study population and ethical consideration}

This study included 100 patients attending the Ministry of health laboratory centers, in El-Fayoum Governorate, these study subjects were randomly selected irrespective of the age-group and both genders were included. All the studied population was informed about the purpose of sample collection and their consents were obtained. Patients were free to refuse sample collection.

\subsection{Study design}

This research is a cross-sectional study designed to evaluate circulating antigen by a commercially available kit (Schistofast ABC Diagnostic, New Damietta city ARE) to determining urinary schistosomiasis in the study population. This study was targeting customers who came for laboratories of health centers for urine analysis. This was performed on 50 positive S.haematobium samples and 50 negative samples for individuals matching in age and sex. This research is a part of the project (Validity of common diagnostic techniques for Schistosoma haematobium infection and evaluation of health centers laboratory results in El-Fayoum Governorate).

\subsection{Collection and processing of urine samples}

Clean specimen bottles were labeled with the needed information and issued to the participating individuals whose informed consent was sought earlier, each patient was given a wide mouth screw- capped container into which to void urine. This was carried out between 10.00 am and $2.00 \mathrm{pm}$ when the ova count of S.haematobium is expected to be at its peak ${ }^{(7)}$. In this study, urinary schistosomiasis was defined as the presence of ova of S.haematobium in the urine.

\subsection{Urine microscopy}

Urine samples were examined for the presence of S.haematobium eggs as in the sedimentation method of Cheesbrough (2006) ${ }^{(8)}$. Each urine sample was mixed thoroughly with a glass rod and three samples were taken each $10 \mathrm{ml}$ urine, one sample for sedimentation centrifugation, another 10 $\mathrm{ml}$ urine sample for Nuclepore membrane filteration technique, and the third sample for circulating antigen in urine detection. The first 10 $\mathrm{ml}$ transferred into a centrifuge tube and centrifuged at $2000 \mathrm{rpm}$ for 5 minutes at room temperature. The supernatant was then discarded and sediment transferred to a microscope glass slide and covered with a coverslip. A drop of Lugol's Iodine was added onto the coverslip prior to examination. The examination of the entire sediment was carried out using the $\mathrm{x} 10$ objective of a compound light microscope.

The second $10 \mathrm{ml}$ urine sample was examined using the Nuclepore membrane filteration technique for S.haematobium eggs detection as in the method of Cheesbrough (2009) ${ }^{(9)}$. The third urine sample was examined using a commercially circulating antigen kit according to the assay procedures.

\subsection{Assay procedure:}

For 100 urine samples, were tested for the presence of circulating antigen by a commercially available kit (Schistofast ABC Diagnostic, New Damietta city ARE). Which were the same kit used by medical laboratories in Egypt), these steps according to company method.

\subsection{Stool microscopy:}


Stool samples were examined for the presence of S.mansoni eggs, only negative stool samples for S.mansoni eggs of 100 population study samples were taken for that research study.

\subsection{Statistical analysis}

Results collected, coded, tabulated, and analyzed through computer facilities using statistical methods S.haematobium infection was defined as any number of eggs greater than zero found in $10 \mathrm{ml}$ of urine, was performed to compare with circulating antigen by commercially kit for Diagnosis of Schistosoma haematobium Infection. Data analysis Correlation of the circulating antigen results with the gold-standard parasitological data was done using diagnostic accuracy tests. In this study, we considered using the sum of the Nuclepore membrane filteration technique and Centrifugation sedimentation technique results as a gold standard to compare them with circulating antigen by commercially kit ${ }^{(10)}$.

\section{Results}

Table (1): shows the Schistosoma haematobium infection as detected by the Circulating antigen in urine related to the intensity of infection detected by parasitologic techniques. In light cases, it detected $54.5 \%$ while in heavy cases it increased to $66.7 \%$. While, P-value is 0.575 not significant at $\mathrm{p}<0.05$.

Table (1): Schistosoma haematobium infection detected by the Circulating antigen in urine relation to the intensity of infection detected by parasitologic techniques.

\begin{tabular}{|c|c|c|c|c|c|c||}
\hline \multirow{2}{*}{\begin{tabular}{c} 
Circulating antigen in $\begin{array}{c}\text { urine } \\
\text { urine }\end{array}$ \\
\cline { 2 - 7 }
\end{tabular}} & \multicolumn{3}{|c|}{$\begin{array}{c}\text { S. haematobium } \\
\text { intensity of infection }\end{array}$} & \multicolumn{2}{c|}{ Total } \\
\cline { 2 - 7 } & No. & $\%$ & No. & $\%$ & No. & $\%$ \\
\hline Negative & 20 & 45.5 & 2 & 33.3 & 22 & 44 \\
\hline Positive & 24 & 54.5 & 4 & 66.7 & 28 & 56 \\
\hline Total & 44 & & 6 & & 50 & \\
\hline
\end{tabular}

*Light infection $=1-49$ eggs per $10 \mathrm{ml}$ of urine.

$* *$ Heavy infection $=50$ or more eggs per $10 \mathrm{ml}$ of urine.

\section{Discussion}

This study showed that circulating antigen detected 4 patients with heavy infected of Schistosoma haematobium from 6 with a sensitivity of $66.7 \%$ while it detected 24 out of 44 patients with light infection of Schistosoma haematobium with a sensitivity of $54.5 \%$. Then the sensitivity of commercial circulating antigen in urine in heavy infected patients was more than the sensitivity in patients with light infection, while $\mathrm{P}$-value is 0.575 not significant at $p<0.05$. The circulating antigen in urine $(\mathrm{CAg})$ by a commercially available kit had a total sensitivity of $56 \%{ }^{(11)}$.

Zeinab A, et al (1995), observed a positive correlation between antigen concentration in either serum or urine samples and egg output ${ }^{(12)}$. Ndhlovu P et al., (1996), studied the relation between the 
intensity of Schistosoma haematobium infection and circulating antigen in people excreting $S$. haematobium eggs. In the group of people excreting from 1 to 50 eggs/10ml (light infection), 81 out of 84 were positive for circulating antigen, giving a sensitivity of $96 \%$. All 12 people excreting more than 50 eggs/10ml (heavy infection), were positive for circulating antigen with a sensitivity of $100 \%$ (13).

Louis et al, (2012), used logistic regression analysis and adjustment to assess the correlation between CCA test and S. haematobium egg counts. The results showed the absence of a correlation between them ${ }^{(14)}$.

Mahfouz A. et al. (2012), found that $S$. haematobium circulating antigen was detected in the urine of 42 out of 50 with a sensitivity of $84 \%$ in the light infection group, and 65 cases out of 70 in heavily infected patients, with a sensitivity of $92.8 \%{ }^{(15)}$. Mahfouz et al., found a significant direct correlation between the level of circulating antigen of Schistosoma haematobium in both serum and urine and the number of eggs excreted in the urine of schistosomiasis patient denoting the reliability of circulating antigen as an indication for the intensity of infection ${ }^{(15)}$. These results were in parallel with those of Hendawy et al., (2006) ${ }^{(16)}$.

The negative results of circulating antigen in urine were found in patients with a low number of egg/10ml urine and this could be due to the possibility that intact ova of $S$. haematobium may release only small undetectable amounts of antigen into the circulation. Another possibility is that the antigen released from the parasite form immune complexes with circulating antibodies (Carlier et al., 1983; Nash 1984) ${ }^{(17,18)}$.

Circulating Schistosome antigen (CSA) disappears rapidly after treatment and can, therefore, be used for assessment of cure. However, the sensitivity of antigen detection varies from 55\% to $100 \%$, being low in low endemic areas (Van
Leishout, 2000) with no advantage over stool and urine examination ${ }^{(3)}$.

\section{Conflict of interest}

There are no conflicts of interest.

\section{Financial support and sponsorship}

This research did not receive any specific grant from funding agencies in the public, commercial, or not for profit sectors.

\section{References:}

1. WHO. 2007. Report of inter country meeting on strategy to eliminate schistosomiasis from the Eastern Mediterranean Region. Muscat, Oman.6-8 November.

2. Ahmed Abdelhalim Yameny. The evolving schistosomiasis agenda 2017- 2020 in Egypt : Moving from control toward final elimination. $\mathrm{J}$ Biosci App Res .2017;3(2): 48- 54.

3. Van Lieshout, L., A. M. Polderman, and A. M. Deelder. 2000. Immunodiagnosis of schistosomiasis by determination of the circulating antigens CAA and CCA, in particular in individuals with recent or light infections. Acta Trop. 77:69-80.

4. Ahmed A. Yameny. Validity of questionnaire to identify Schistosoma haematobium infection. J Biosci App Res .2017;3(4): 221- 228.

5. Ahmed A. Yameny. The Validity of Haematuria and Proteinuria by chemical reagent strip for diagnosis of Schistosoma haematobium infection. J Biosci App Res .2017;3(4): 229- 236.

6. Deelder AM, Klappe HT, van den Aardweg GJ, van Meerbeke EH (1976) Schistosoma mansoni: demonstration of two circulating antigens in infected hamsters. Exp Parasitol 40: 189-197.

7. Eyong ME, Ikepeme EE, Ekanem EE. Relationship between Schistosoma haematobium infection and urinary tract infection among children in South Eastern, Nigeria. Niger Postgrad Med J2008;15:89-93. 
8. Cheesbrough M. District laboratory practice in Tropical countries part 2. United Kingdom :Cambridge University Press. Cambridge. 2006; 323-41.

9. Cheesbrough M. Laboratory-practice-intropical-countries-part-1. United Kingdom :Cambridge University Press. Cambridge. 2009;p.357

10. Ahmed A. Yameny. Evaluation of Nuclepore Filteration Technique for Diagnosis of Schistosoma haematobium Infection.J Biosci App Res. 2015;1(6):335-341.

11. Ahmed Abdelhalim Yameny.Evaluation of circulating antigen in urine technique for diagnosis of Schistosoma haematobium infection. J Biosci App Res. 2018;4(4):519525.

12. Demerdash ZA, Mohamed SH,Shaker ZA, Hassan SI, El attar GM, Saadel din AA, et al.Detection of circulating schistosome antigens in serum and urine of schistosomiasis patients and assessment of cure by a monoclonal antibody.J. Egypt. Soc. Parasitol.,25(2) 1995; 471 - 484.

13. Ndhlovu P,Cadman H,Gundersen S,Vennervald BJ,Friis $\mathrm{H}$, Christensen NO.et al Circulating Anodic Antigen (CAA) levels in different age groups in a Zimbabwean rural community endemic for schistosoma haematobium determined using the magnetic beads Antigen- capture
Enzyme -Linked Immunoassay. Am J Trop Med Hyg . 54(5),1996, pp. 537- 542 .

14. Tchuenté L T, Fouodo C K, Ngassam RK, Sumo L, Noumedem CD, Kenfack CM, et al .Evaluation of Circulating Cathodic Antigen (CCA) Urine-Tests for Diagnosis of Schistosoma mansoni Infection in Cameroon July 2012 Issue of PLoS Neglected Tropical Diseases

15. Mahfouz A., Mahana N., Rabee I.and El Amir A., 2012. Evaluation of Different Immunological Techniques for Diagnosis ofSchistosomiasis haematobium in Egypt. Biotechnology, 11: 10-19.

16. Hendawy, M.A., S.H. Mohamed and R.A. Ibrahim, 2006. Nitrocellulose cassettes (NCC) as a field applicable technique for rapid and effective diagnosis of schistosomiasis and fascioliasis. New Egypt J. Med., 35: 86-92.

17. Carlier, Y., H. Nzeyimana, D. Boul and A. Capron, 1983. Evaluation of circulating antigens by a sandwich radioimmunoassay and of antibodies and immune complex, in Schistosoma mansoni-infected African parturients and their newborn children. Am. J. Trop. Med. Hyg., 29: 74-81.

18. Nash, T.E., 1984. Immune complex size for determines the clearance rate of circulating antigen in schistosome infected mice. Am. J. Trop. Med. Hyg., 33: 621-626. 